\title{
GAUSSIAN FLUCTUATIONS OF MOLECULAR FIELD IN QUASI-ONE-DIMENSIONAL ISING MODEL
}

\author{
Z. ONYSZKIEWICZ ${ }^{a}$ AND A. WIERZBICKI ${ }^{b}$ \\ ${ }^{a}$ Magnetism Theory Division, Faculty of Physics, A. Mickiewicz University \\ 61-614 Poznań, Poland \\ ${ }^{b}$ Department of Chemistry, University of South Alabama \\ Mobile, Alabama, 36688 USA
}

(Received November 13, 1998)

\begin{abstract}
Quasi-one-dimensional spin systems described by an Ising-like Hamiltonian with a strong space anisotropy $(s=1 / 2)$ are investigated. Magnetic properties of this model are examined in the approximation including Gaussian fluctuations of molecular field. This paper reports an attempt at obtaining more accurate results for Gaussian fluctuation of molecular field by an exact formula for mean fluctuations of a. spin.
\end{abstract}

PACS numbers: $75.10 . \mathrm{Hk}, 75.70 . \mathrm{Ak}$

\section{Introduction}

Studies of low-dimensional or quasi-low-dimensional (QLD) spin systems are characterized by a close coupling between theory and experiment because of the rapidly increasing number of experimental works on real materials with quasi-low-dimensional structures and properties [1]. These materials often correspond remarkably close to certain idealized one- or two-dimensional spin models, and are great challenges not only for physicists, but also for chemists and technologists. Low-dimensional magnetic systems have always been very attractive from the theoretical point of view. Many non-trivial models are soluble in one or two dimensions, while they are only approximately understood in three dimensions. Therefore, the exact solution serves as a useful test of approximate mathematical methods, and certain features of the low-dimensional solution remain relevant in higher dimensions.

In this paper we shall consider only magnetic properties of quasi-one-dimensional (Q1D) magnetic systems which are well described by the simple models of 
localized and ordered spins with the following Ising-like Hamiltonian with a strong space anisotropy $(s=1 / 2)$

$$
H=-\frac{1}{2} \sum^{\prime} S_{\boldsymbol{f}}^{z}\left(J_{1} S_{\boldsymbol{f}^{\prime}}^{z}+J_{2} S_{\boldsymbol{f}^{\prime \prime}}^{z}\right)-g \mu_{\mathrm{B}} B \sum_{\boldsymbol{f}} S_{\boldsymbol{f}}^{z},
$$

where $J_{1}$ and $J_{2}$ are coupling parameters in the $f-f^{\prime}$ and $f-f^{\prime \prime}$ directions, respectively, and $\sum^{\prime}$ stands for summation over pairs of different lattice points. In particular for Q1D systems we have

$$
\left|J_{1}\right| \gg\left|J_{2}\right|
$$

where $J_{1}$ is the intra-chain coupling parameter. The symbol $B$ is the external magnetic field in the $z$ direction.

The aim of our paper is to give a detailed analysis of the magnetic properties of the Q1D spin model described by Hamiltonian (1), performed in the modified Gaussian fluctuations approximation (MGFA) which has been proposed in the communication [2] and paper [3]. In paper [3] it has been shown that MGFA can be used for description of the quasi-two-dimensional spin systems. A comparison with the exact results will test the suitability of the MGFA for description of Q1D spin systems. In this work we will show that MGFA can be used for description of the magnetic and thermal properties of Q1D spin systems provided that the choice of the parameters of the theory is made taking into regard the size of mean inhomogeneous fluctuations of the molecular field.

This paper reports an attempt at obtaining more accurate results within Gaussian fluctuations approximation for Q1D spin systems by replacing the expansion based on classification of the Feynman diagrams with respect to powers of the parameter $1 / z$, where $z$ is the effective number of spins interacting with any given spin, by an exact formula for mean fluctuations of a spin. The formulation of MGFA allows us to dispose of certain veiled assumptions concerning the interspin correlations taken in Ref. [4].

\section{Modified Gaussian fluctuations approximation}

As a starting point of the modified MGFA [2-3] we choose the following decomposition of the Hamiltonian (1):

$$
H=\left(H-H_{1}\right)+H_{1}=H_{0}+H_{1},
$$

where the perturbative part $H_{1}$ is defined by the transformation

$$
\begin{aligned}
& H \rightarrow H_{1}=H\left(S_{f}^{z} S_{f^{\prime}}^{z} \rightarrow 0, S_{f}^{z} S_{f^{\prime \prime}}^{z} \rightarrow \delta S_{f}^{z} \delta S_{f^{\prime \prime}}^{z}\right), \\
& \delta S_{f}^{z}=S_{f}^{z}-\left\langle S^{z}\right\rangle, \delta S_{f^{\prime \prime}}^{z}=S_{f^{\prime \prime}}^{z}-\left\langle S^{z}\right\rangle, \\
& \left\langle S^{z}\right\rangle=\operatorname{Tr}\left\{S^{z} \exp [\beta(F-H)]\right\}, \quad \beta=\left(k_{\mathrm{B}} T\right)^{-1}
\end{aligned}
$$

and

$$
F=-\frac{1}{\beta} \ln \operatorname{Tr}[\exp (-\beta H)]
$$

is the free energy. 
Hence, we have the following decomposition of the Hamiltonian (1)

$$
\begin{aligned}
& H_{0}=\frac{1}{2} N z J_{2}\left\langle S^{z}\right\rangle^{2}-\sum_{\boldsymbol{f}} S_{\boldsymbol{f}}^{z}\left(\frac{1}{\beta} y+\sum_{f^{\prime}} J_{1} S_{\boldsymbol{f}^{\prime}}^{z}\right), \\
& H_{1}=-\frac{1}{2} \sum^{\prime} \delta S_{f}^{z} J_{2} \delta S_{f^{\prime \prime}}^{z}
\end{aligned}
$$

where

$$
\begin{aligned}
& y=y\left(\left\langle S^{z}\right\rangle\right)=\beta\left[g \mu_{\mathrm{B}} B+z J_{2}\left\langle S^{z}\right\rangle\right], \\
& z=\sum_{f^{\prime \prime}}
\end{aligned}
$$

and $N$ is the number of spins in the lattice.

Applying Eqs. (7) and (8) we obtain the free energy in the linear chain approximation (LCA) [5]

$$
F_{0}=-\beta^{-1} \ln \operatorname{Tr}\left[\exp \left(-\beta H_{0}\right)\right]=N\left(\frac{1}{2} z J_{2}\left\langle S^{z}\right\rangle^{2}-\beta^{-1} L\right),
$$

where

$$
L=\ln \left[\cosh \left(\frac{1}{2} y\right)+\left(\sinh ^{2}\left(\frac{1}{2} y\right)+\exp \left(-\beta J_{1}\right)\right)^{1 / 2}\right]+\frac{1}{4} \beta J_{1} .
$$

Equation (11) approximates the exact free energy $F$ of model (1) by the free energy of noninteracting Ising chains placed in a molecular field (10).

In the proposed approach we assume that the free erergy of our model $\bar{F}_{0}$ can be found from the Gaussian distribution as the free LCA energy a veraged over molecular field fluctuations, expressed by Eq. (11). This value is

$$
\bar{F}_{0}=\frac{1}{\sqrt{2 \pi}} \int_{-\infty}^{+\infty} \exp \left(-\frac{1}{2} x^{2}\right) F_{0}(x) \mathrm{d} x
$$

where

$$
F_{0} \rightarrow F_{0}(x)=F_{0}\left(y\left(\left\langle S^{z}\right\rangle\right) \rightarrow y\left(\left\langle S^{z}\right\rangle+\delta\left\langle S^{z}\right\rangle x\right)\right)
$$

and where $\delta\left\langle S^{z}\right\rangle$ is the average Gaussian fluctuation of the $\left\langle S^{z}\right\rangle$.

As follows from Eq. (7), the free energy $F$ does not fluctuate, however, we can assume that $F_{0}$ fluctuates as this is only an approximate of the free energy of the studied system. Thus, the assumption expressed by Eq. (12) is justified on condition that the free energy $\bar{F}_{0}$ satisfies the following stationary conditions:

$$
\begin{gathered}
\frac{\partial \bar{F}_{0}}{\partial\left\langle S^{z}\right\rangle}=0, \\
\frac{\partial \bar{F}_{0}}{\partial \delta\left\langle S^{z}\right\rangle}=0 .
\end{gathered}
$$

From (13) we get

$$
\left\langle S^{z}\right\rangle=-\frac{1}{g \mu_{\mathrm{B}} N} \frac{\partial \bar{F}_{0}}{\partial B}=\frac{\partial \bar{L}}{\partial y},
$$


where

$$
\bar{L}=\frac{1}{\sqrt{2 \pi}} \int_{-\infty}^{+\infty} \exp \left(-\frac{1}{2} x^{2}\right) L(y+\delta y x) \mathrm{d} x
$$

and

$$
\delta y=\beta z J_{2} \delta\left\langle S^{z}\right\rangle
$$

is the average Gaussian fluctuations of the molecular field.

As follows from (14), the free energy $\bar{F}_{0}$ is stationary for any finite average fluctuations $\delta\left\langle S^{2}\right\rangle$, i.e., for $\left|\delta\left\langle S^{z}\right\rangle\right|<\infty$. Therefore, the condition (14) cannot be used for determination of $\delta\left\langle S^{2}\right\rangle$. However, we can do it with the help of the exact identity

$$
\left\langle\delta S^{z}\right\rangle=0
$$

following from Eq. (5). Having calculated the derivative of (18) with respect to $y$

$$
\frac{\partial\left\langle\delta S^{z}\right\rangle}{\partial y}=\frac{\partial}{\partial y}\left\langle S^{z}-\frac{\partial \bar{L}}{\delta y}\right\rangle=0
$$

we have

$$
\sqrt{\left\langle\left(\delta S_{f}^{z}\right)\right\rangle}=\sqrt{\frac{1}{N} \frac{\partial^{2} \bar{L}}{\partial y^{2}}}
$$

As follows from (20)

$$
\lim _{N \rightarrow \infty} \delta\left\langle S^{z}\right\rangle=0
$$

To avoid the disappearance of $\delta\left\langle S^{z}\right\rangle$ with increasing number of spins in a lattice $N$, we assume that (see paper [3] for more details)

$$
\delta\left\langle S^{z}\right\rangle=\sqrt{\frac{1}{\widetilde{N}} \frac{\partial^{2} \bar{L}}{\partial y^{2}}}
$$

where $\tilde{N}$ is the number of Ising chains making a cluster (with periodical boundary conditions) of the size corresponding to the size of the averaged inhomogeneous fluctuation of the molecular field. In our approach $\tilde{N}$ is a parameter of the theory.

Substituting Eq. (22) to (17) we obtain the following expression for the average Gaussian fluctuations of the molecular field:

$$
\delta y=\beta z J_{2} \sqrt{\frac{1}{\widetilde{N}} \frac{\partial^{2} \bar{L}}{\partial y^{2}}} .
$$

Equations (12), (15) and (23) make a closed set of equations enabling calculating of the mean value of the spin $\left\langle S^{z}\right\rangle$ and its averaged Gaussian fluctuation $\delta\left\langle S^{z}\right\rangle$. The proposed approach is based on the two assumptions: concerning the choice of the free energy expression as a Gaussian average (12), and natural assumptions as to the average Gaussian fluctuations of the molecular field $\delta y$. 


\section{Numerical results}

In order to carry out the calculations we have specified the magnetic lattices as a honeycomb, square and triangular. Equations (15) and (23) have been solved numerically assuming the value of $\widetilde{N}=3,4,5, \ldots$, appropriate for a given $J_{2} / J_{1}$ and number of nearest neighbours in a given lattice. The results are presented graphically. Figure 1 shows the relative Curie temperature $t_{\mathrm{C}}=k_{\mathrm{B}} T_{\mathrm{C}} / J_{1}$ versus the anisotropy $J_{2} / J_{1}$, as obtained within the molecular field approximation (MFA), linear chain approximation (LCA) [5] (for $\widetilde{N}=\infty$ ), and the approximation assumed in the present paper (MGFA) as well as the exact results for a square lattice (sq). For $J_{2} / J_{1}=1$ we obtained in MGFA for a square lattice $t_{\mathrm{C}}=0.584532$, while the exact value is $t_{\mathrm{C}}=0.567296$ [7].

The plots $t_{\mathrm{C}}=t_{\mathrm{C}}\left(J_{2} / J_{1}\right)$ calculated in MGFA have the broken lines (- - ) as in this approximation below certain values of $J_{2} / J_{1}$, the Curie point becomes only the lower limit of the paramagnetic phase existence due to the appearance of a tricritical point. This is a defect of the MGFA as the exact calculations do not indicate the existence of this point. The tricritical point appears also for other lattices, as shown in Fig. 2. The tricritical point temperature for all lattices studied is a decreasing function of $\widetilde{N}$. This unphysical tricritical point can be eliminated by assuming an appropriate value of $\widetilde{N}$ for a certain ratio of $J_{2} / J_{1}$. As follows from Fig. 1, for a given $\widetilde{N}$ (for a square lattice $\widetilde{N} \geq 3$ ), there is a $J_{2} / J_{1}<1$, for which $t_{\mathrm{C}}$ calculated in MGFA is equal to the exact value of the Curie point.

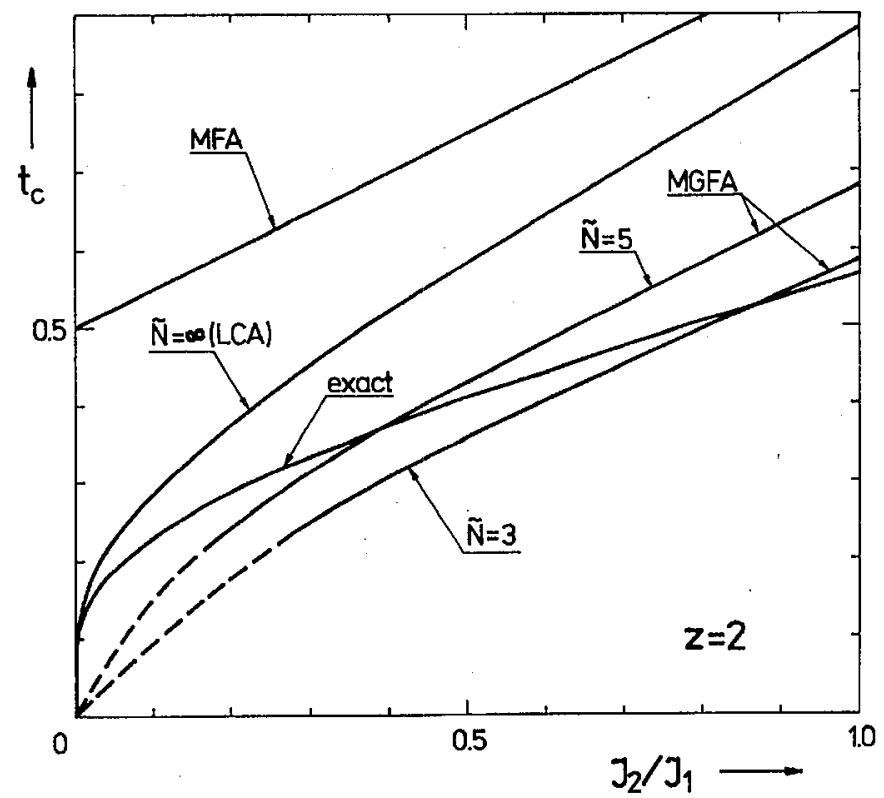

Fig. 1. Variation of the Curie temperature $t_{C}$ (in relative units) with space anisotropy $J_{2} / J_{1}$ for MFA, LCA, exact [7] and MGFA (the present paper) for square (sq) lattice. 

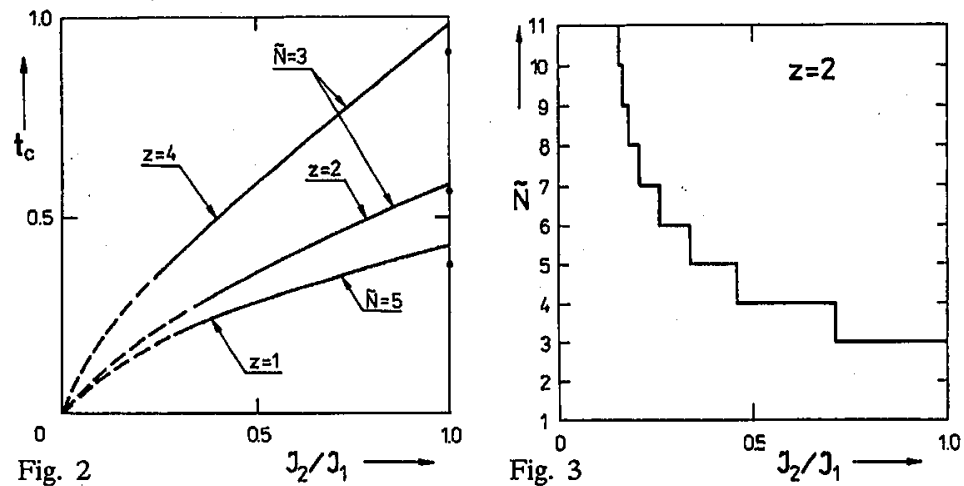

Fig. 2. Dependence Curie temperature $t_{\mathrm{C}}$ (in relative units) on space anisotropy $J_{2} / J_{1}$ for honeycomb lattice $(z=1)$, square lattice $(z=2)$ and triangular lattice $(z=4)$. Exact results are given by dots [6].

Fig. 3. Optimal value of the parameter $\tilde{N}$ versus space anisotropy $J_{2} / J_{1}$ for square lattice.

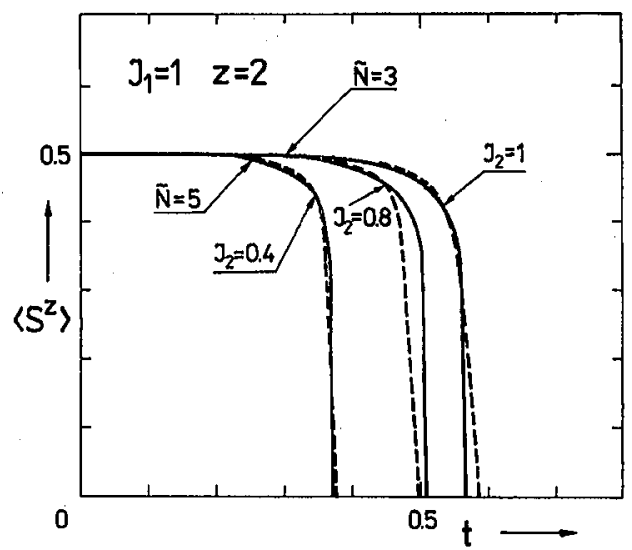

Fig. 4. The dependence of relative magnetization $\left\langle S^{z}\right\rangle$ on relative temperature $t$ for different space anisotropies. $J_{2} / J_{1}$ obtained in MGFA (- - ) and exact results (-) [7] for square lattice.

Depending on the value of the ratio $J_{2} / J_{1}$, we can choose the optimum value of $\tilde{N}$. An example of such an optimum choice of $\tilde{N}$ (for a square lattice) is shown in Fig. 3. The diagram $\widetilde{N}=\widetilde{N}\left(J_{2} / J_{1}\right)$ obtained is the so-called "devil steps". As the parameter $\widetilde{N}$ determines the size of inhomogeneous fluctuations of the molecular field (see expression (23)) on the basis of Fig. 3, we can conclude that with increasing spatial anisotropy - so with decreasing $J_{2} / J_{1}$ ratio - the average size of inhomogeneous fluctuations of the molecular field increases. In the limiting case of $J_{2} / J_{1} \rightarrow 0$, we have to assume that $\widetilde{N} \rightarrow \infty$, which corresponds to the LCA approximation.

The above results indicate that the optimum choice of $\widetilde{N}$ in investigation of more complex spin systems within MGFA should not pose considerable problems. 

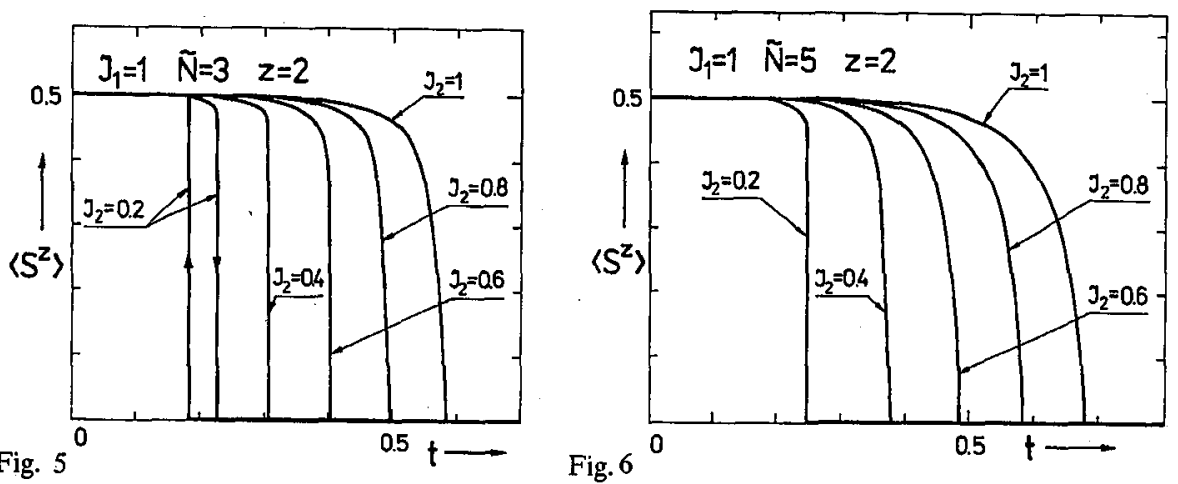

Fig. 5. Relative spontaneous magnetization $\left\langle S^{z}\right\rangle$ versus temperature $t$, calculated for different space anisotropy $J_{2} / J_{1}$ and $\widetilde{N}=3$.

Fig. 6. Spontaneous magnetization $\left\langle S^{z}\right\rangle$ versus temperature $t$ for square lattice for $\widetilde{N}=5$.
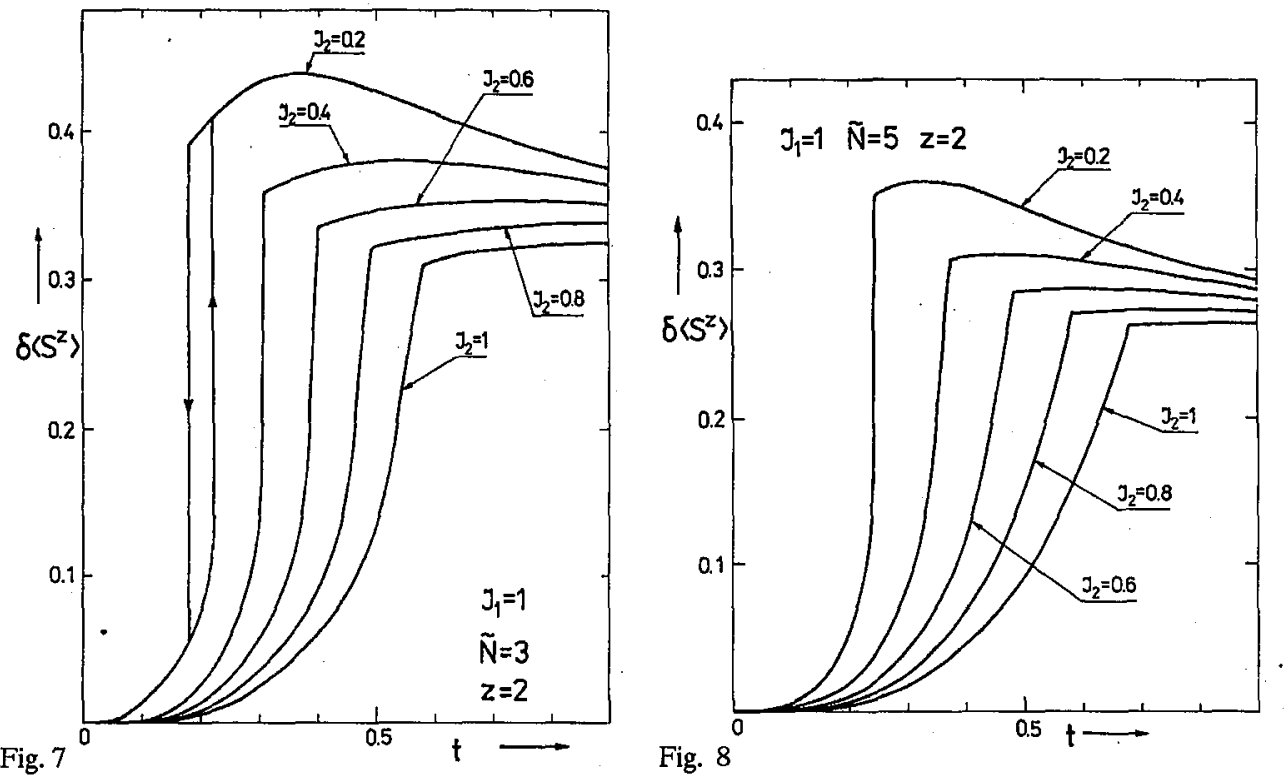

Fig. 8

Fig. 7. Dependence of the average Gaussian fluctuation $\delta\left\langle S^{z}\right\rangle$ (in relative units) on temperature $t$ for different $J_{2} / J_{1}$ and for sq lattice.

Fig. 8. Variation of Gaussian average fluctuation $\delta\left\langle S^{z}\right\rangle$ (in relative units) with temperature $t$ for different $J_{2} / J_{1}$ and for sq lattice.

Figure 4 presents a comparison of the temperature dependence $\left(t=k_{\mathrm{B}} T / J_{1}\right)$ of the mean spin moment, proportional to magnetization, calculated for a square lattice in MGFA with the exact results [7, 8].

Figures 5-10 present the temperature dependence of relative magnetization $\left\langle S^{z}\right\rangle$, Gaussian average fluctuations $\delta\left\langle S^{z}\right\rangle$ of relative magnetization and average Gaussian fluctuations of molecular field $\delta y$ for different values of space anisotropy 


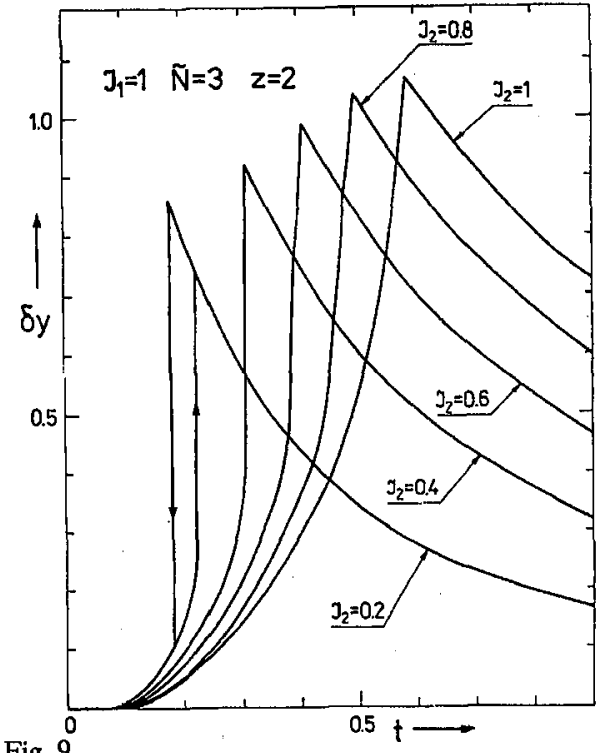

Fig. 9

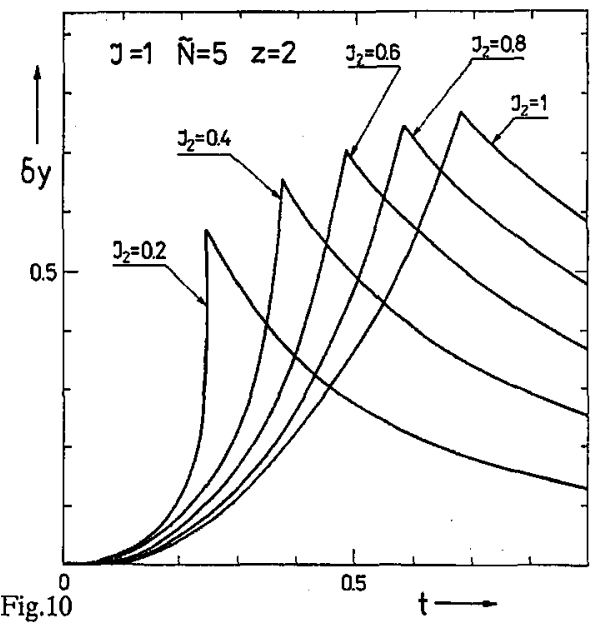

Fig. 9. Gaussian average fluctuation $\delta y$ of the molecular field against temperature $t$ for different space anisotropy $J_{2} / J_{1}$ and $\widetilde{N}=3$ (square lattice).

Fig. 10. Temperature dependence of the average Gaussian fluctuation $\delta y$ of molecular field for sq lattice and for different space anisotropy $J_{2} / J_{1}$.

$J_{2} / J_{1}$ for a square lattice. These figures show the hysteresis loops which result from the appearance of the tricritical points which do not appear in the exact solutions below which there are discontinuous phase transitions from the ferromagnetic to paramagnetic phase.

\section{Conclusions}

On the basis of the results obtained in the reported work we can draw the following conclusions:

(i) under a proper choice of $\widetilde{N}$, MGFA permits a correct description of the properties of Q1D Ising like models for an arbitrary space anisotropy $J_{2} / J_{1}$. The values of the Curie point $t_{\mathrm{C}}$ calculated in MGFA for certain values of the ratio $J_{2} / J_{1}$ and for the appropriate choice of $\widetilde{N}$, are equal to the exact $t_{\mathrm{c}}$ values, which means that despite its formal simplicity, MFGA can be an effective and accurate method for description of Q1D spin systems.

(ii) The MGFA can be easily generalised onto multisublattice spin systems of the spin $s>1 / 2$.

(iii) A relative simplicity and high accuracy of the MGFA make it a recommendable efficient tool for investigation of Q1D magnetic systems of complex magnetic structure for which exact solutions are not known, such as e.g. metamagnetics characterised by a strong spatial anisotropy.

(iv) The modified MGFA can be successfully used for investigation of such thermal properties of spin systems as entropy and heat capacity. 


\section{Acknowledgments}

This work was supported by the project 8T11F01509 of the Committee for Scientific Research.

\section{References}

[1] Proc. Intern. Conf. on Magnetism, Warsaw 1994, Part III, North-Holland, Amsterdam 1995, Ch. 21.

[2] Z. Onyszkiewicz, J. Magn. Magn. Mater. 139, L3 (1995).

[3] Z. Onyszkiewicz, A. Wierzbicki, J. Magn. Magn. Mater. 174, 174 (1997).

[4] Z. Onyszkiewicz, Phys. Lett. 124, 485 (1987).

[5] I.A. Plascak, N.P. Silva, Phys. Status Solidi A 10, 669 (1982).

[6] A. Toda, R. Kubo, A. Saito, Statistical Physics I. Equilibrium Statistical Mechanics, Springer, Berlin 1983.

[7] C.N. Yang, Phys. Rev. 85, 808 (1952).

[8] A. Lipowski, M. Suzuki, J. Phys. Soc. Jpn. 61, 4356 (1992). 